\title{
Mechanical properties evaluation of sisal fibre reinforced polymer composites: a review
}

\begin{abstract}
ABSTRCT
Recently, growing environmental impact associated with production, disposal and recycling of synthetic fibre based polymer composites triggers the development of ecofriendly composite for various applications such as automotive, marine, chemical, Infrastructure, sporting goods etc. Among many natural fibres like kenaf, jute, oil palm, cotton, flax, banana and hemp, sisal are gaining attention as they are abundantly available, cheaper, eco-friendly and possess remarkable and satisfactory mechanical properties to hemp, banana and jute. Sisal fibre will play a key role to fabricate a varied range of structural and non-structural industrial products with different polymer matrix. This review article deals the mechanical properties of sisal fibre and the several factors influencing the mechanical properties of its polymer composites, such as fibre loadings, fibre length, fibre architecture, chemical treatments and hybridization by incorporating different natural/synthetic fibre/fillers or additive, according to the application and strength requirements. Attempt also been made to investigate the effect of water absorption, chemical concentration, exposure time, filler weight $\%$ and individual fibre loading $\%$ in the hybrid configuration on the mechanical properties. Overall present review article was designed to explore, highlights and gathered the previous reported studies directing the mechanical properties of sisal fibre and its polymer composites to provide a perfect source of data and literature for doing future research to reveal it as construction and building materials like synthetic fibres.
\end{abstract}

Keyword: Natural fibres; Sisal fibre; Sisal fibre reinforced polymer composites; Mechanical properties; Chemical treatments; Hybrid composites. 